\title{
Análisis comparativo de la bibliografía que utilizan los investigadores del Instituto de Investigaciones en Matemáticas Aplicadas y en Sistemas
}

\author{
Comparative analysis of the literature used by researchers of IIMAS
}

\section{Suyin Ortega Cuevas (1) y Leticia López Huerta (2)}

Instituto de Investigaciones en Matemáticas Aplicadas y en Sistemas. Universidad Nacional Autónoma de México. México D. F. CP. 04510.(1) suyin@leibniz.iimas.unam.mx (2) Ilopez@leibniz.iimas.unam.mx.

\begin{abstract}
Resumen
Se realiza un análisis comparativo del desarrollo de la colección de la Biblioteca del Instituto de Investigaciones en Matemáticas Aplicadas y en Sistemas (IIMAS), tomando como base las referencias bibliográficas que utilizaron los investigadores del IIMAS en los artículos publicados durante el año de 2007.
\end{abstract}

Palabras clave: Análisis de referencias. Análisis de desarrollo de colecciones. Bibliometría. Instituto de Investigaciones en Matemáticas Aplicadas y en Sistemas (IIMAS). México.

\section{Introducción}

El desarrollo de colecciones se ha enfocado a la satisfacción de las necesidades de información de los usuarios. El adquirir material bibliohemerográfico es la prioridad número uno en las bibliotecas. Sin embargo, es imposible comprar todo lo que se necesita, puesto que no hay presupuesto suficiente para satisfacer todas las necesidades de información de la comunidad a la que se le proporciona el servicio; motivo por el cuál se debe realizar un análisis de las obras que pueden ser adquiridas, evaluando el acervo, conforme a las políticas de la institución y las necesidades de los mismos usuarios, y buscando nuevo material a través de catálogos, portales, librerías entre otros, para de esta manera cumplir con el objetivo de cualquier unidad de información. En teoría, una biblioteca académica que apoya trabajos de investigación debe tener el material bibliohemerográfico que los investigadores solicitan, pero en la práctica, surgen varias preguntas: ¿Se cumplirá? ¿Cuánta información encuentran los usuarios dentro de su misma biblioteca? ¿Se les proporciona lo que buscan? ¿Se adquirirá un porcentaje mayor al $50 \%$ de lo que los usuarios solicitan? ¿Por qué el $50 \%$ ? (Por considerar que si se tiene más

\begin{abstract}
A comparative analysis of the development of the library collection of the Instituto de Investigaciones en Matemáticas Aplicadas y en Sistemas (IIMAS) was carried out, based on the references used by the IIMAS researchers in articles published during the year 2007.
\end{abstract}

Keywords: Bibliographic reference analysis. Collection development analysis. Bibliometrics. Instituto de Investigaciones en Matemáticas Aplicadas y en Sistemas (IIMAS). México.

de la mitad que se busca se está cumpliendo con la función).

La Biblioteca del IIMAS, única en su colección, siempre está al pendiente de adquirir lo más nuevo, lo más relevante, y en ocasiones material retrospectivo. También suscribe títulos de revistas que los investigadores consideran importantes para el apoyo de sus pesquisas y para apoyar a los posgrados que se imparten en el Instituto. Sin embargo al no tener una certeza documentada de si realmente se cumple con esta misión, surge la inquietud de realizar una evaluación cuantitativa a través de los artículos que se publican en revistas arbitradas la comunidad académica del Instituto de Investigaciones en Matemáticas Aplicadas y en Sistemas (IIMAS).

\section{El Instituto de Investigaciones en Matemáticas Aplicadas y en Sistemas}

El Instituto de Investigaciones en Matemáticas Aplicadas y en Sistemas (IIMAS), tiene como antecedente al Centro de Cálculo Electrónico (CCE) que se funda en el año 1958, dentro de la Facultad de Ciencias. En ese mismo año se instaló en el Centro la primera computadora en México y en Latinoamérica, con el fin de utilizar- 
la para el avance de la ciencia. Además, se enviaron estudiantes al extranjero para cursar posgrados en la nueva área del conocimiento. A partir 1967 el Centro se moderniza, se adquiere una computadora con tecnología mas avanzada y se cuenta con personal especializado y el número de usuarios aumenta de 60 a 2000. La evolución del CCE llevó en el año 1970 a transformarlo en el Centro de Investigaciones en Matemáticas Aplicadas, Sistemas y Servicios (CIMASS) e inicia formalmente sus actividades de investigación en cómputo y en estadística.

En 1973, el CIMASS se dividió en dos centros, el de Servicios de Cómputo y el de Investigaciones en Matemáticas Aplicadas y en Sistemas (CIMAS). Se amplían los grupos de trabajo y diversifican las actividades. En 1976 se aprueba su conversión en Instituto de Investigaciones en Matemáticas Aplicadas y en Sistemas (IIMAS).

Actualmente, el IIMAS forma parte del Subsistema de la Investigación Científica, es una de las doce entidades académicas que conforman el área de investigación en ciencias físicomatemáticas y está formado por seis departamentos académicos agrupados en dos áreas: el Área de Matemáticas Aplicadas y Sistemas, integrada por cuatro departamentos -Matemáticas y Mecánica, Métodos Matemáticos y Numéricos, Modelación Matemática en Sistemas Sociales, y, por último, Probabilidad y Estadística-; y el Área de Ciencia e Ingeniería de la Computación, formada por dos departamentos -Ciencias de la Computación e Ingeniería de Sistemas Computacionales y Automatización.

El IIMAS también participa en los Posgrados con sede en el IIMAS -Ciencias Matemáticas y de la Especialización en Estadística Aplicada; Ciencia e Ingeniería de la Computación- y colabora en los Posgrados en Ingeniería y Ciencias de la Tierra.

\section{La Biblioteca IIMAS}

La labor del Instituto contribuye de manera importante en la presencia que tiene la UNAM a nivel nacional e internacional en el ámbito de la investigación. La producción científica del Instituto se manifiesta principalmente en los artículos publicados en revistas de circulación internacional arbitradas. Para ello, el apoyo que brinda la Biblioteca IIMAS es fundamental. Actualmente la Biblioteca está organizada en tres áreas: Servicios al Público, Servicios Especializados y Servicios Técnicos, coordinadas por una Jefatura.
Para el desarrollo de colecciones, el área de Servicios Especializados trabaja muy de cerca con el usuario, y se encarga de seleccionar y difundir recursos digitales especializados, así como de dar a conocer las últimas novedades bibliográficas, procurando ofrecer títulos que no estén disponibles en otras bibliotecas.

Por su parte, el área de Servicios Técnicos es la responsable de administrar el presupuesto asignado para la adquisición del material bibliohemerográfico y se encarga de contactar a proveedores que cumplan con las expectativas del Instituto. Actualmente, el presupuesto asignado para la compra de libros cubre la demanda de nuestros investigadores, en promedio se adquieren 650 títulos al año. La suscripción de revistas es otra historia: desde el año 1996, el presupuesto ha sido insuficiente para sostener en promedio 360 suscripciones de revistas; y se han tenido que buscar soluciones que no afecten la colección, como incrementos en el presupuesto, acceso libre a revistas electrónicas, e incluso la participación en el Proyecto de Racionalización de Revistas (PRR) promovido por la Dirección General de Bibliotecas (DGB), dependiente de la Universidad Nacional Autónoma de México (UNAM). Este proyecto fue importante porque fue posible tener estudio mas claro de los títulos duplicados en bibliotecas del área de la investigación científica de la UNAM y también confirmar la importancia de la Biblioteca IIMAS, ya que posee una colección única de revistas en su especialidad a nivel nacional y quizá de Latinoamérica.

La Biblioteca IIMAS es consciente de los altos costos de la suscripción a revistas, siendo imposible suscribir la totalidad de ellas, por lo que se apoya del Sistema Bibliotecario de la UNAM, que cuenta con 140 bibliotecas en todos sus niveles y sedes - tanto locales como foráneas-, en las que es posible localizar gran parte de la información que los investigadores solicitan a través del préstamo interbibliotecario, principalmente con el subsistema de la Investigación Científica, la Coordinación de Humanidades y las facultades o bien -como última opción- la solicitud de artículos a la British Library.

\section{Metodología}

Cada año, el Instituto publica un informe de actividades en donde se desglosan de manera detallada lo que se realizó durante el año anterior. En dicho informe, se tienen los datos de los artículos en revistas arbitradas, libros, artículos y capítulos en libros, artículos en memorias con arbitraje, artículos en memorias sin arbitraje, artículos y entrevistas en medios de divulgación, 
entrevistas y programas de docencia y divulgación, publicaciones del IIMAS, preimpresos y reportes de investigación en dónde participa el personal académico. De aquí, se ha utilizado tan sólo la información exclusivamente de las publicaciones arbitradas, siendo este nuestro objeto de estudio.

Una vez que se obtuvo el listado, se procedió a la tarea de obtener los 77 artículos que participan en el estudio. El primer filtro de obtención fue localizar aquellos que se encontraban disponibles en formato electrónico (47), sobrando 30 documentos. El segundo filtro fue buscarlos en formato impreso a través de las 140 bibliotecas que integran el Sistema Bibliotecario de la UNAM, obteniendo 17. De los 13 restantes, se busco directamente al autor; y solo se obtuvieron 3 artículos. Los 10 restantes no fue posible rastrearlos. El motivo fue que el autor estaba fuera del Instituto o bien no se pudo hacer el contacto.

Considerando que se tenía más del $80 \%$ de los artículos, se optó por realizar el análisis.

Una vez que se obtuvo la información, se procedió a realizar una base de datos en dónde se concentró la información para poder ser manipulada, analizando los resultados estadísticos. EI mayor problema que se identificó fue la uniformidad en la colocación de los datos bibliográficos. Cada editor tiene sus propias normas bibliográficas variando de uno a otro y el problema de la normalización se hizo patente.

La base de datos constó de 1565 referencias. Primeramente, se identificaron cuales eran referencias de libros, artículos en revistas, normas, sitios Web, tesis y una variable que no era considerada: los colegios invisibles. Una vez separada la información, se consultaron las bases de datos de LIBRUNAM, TESIUNAM y SERIUNAM para localizar si las referencias estaban dentro del acervo de la Universidad en primera instancia; y, en segunda, si es acervo del IIMAS. En tercer lugar, se comprobó si se encontraban en formato electrónico en la Biblioteca Digital (BIDI) de la UNAM.

Con las revistas se hizo un análisis más detallado, puesto que el hecho de aparecer el título en los catálogos consultados se verificó que el número consultado se tuviera disponible ya fuera en formato electrónico o bien de manera impresa, por lo que hubo títulos que si estaban registrados pero si el número que daba la referencia no estaba registrado se daba como no disponible.
Una vez que la base de datos estuvo completa y la información uniformada, se procedió a extraer los datos para ser analizados.

La metodología utilizada fue analítica, puesto que es un análisis comparativo. Se escogió 2007 por ser el año inmediato anterior, considerado el más relevante por el momento.

\section{Análisis e interpretación de los resultados}

La figura 1, representa de manera gráfica el porcentaje de referencias localizadas. De las 77 , se localizaron el $87 \%$ que es igual a 67 artículos. Por tratarse de más del $50 \%$, se concluye que es un estudio altamente representativo.

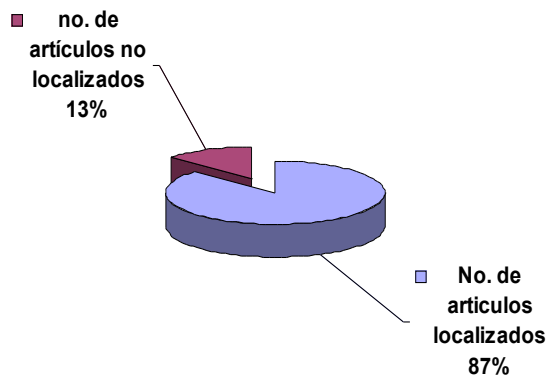

Figura 1. Total de referencias localizadas

La figura 2 se refiere a los tipos de documentos por categoría. Se obtuvo que los artículos en revistas son los más consultados, alcanzando el número de 1106, siendo más de la mitad del universo. En segundo lugar, se ubican los libros con un porcentaje también representativo del $27 \%$; las tesis tienen cerca del $1 \%$ al igual que los colegios invisibles; los sitios Web también se hacen notar, aunque todavía en un porcentaje menor; y las normas casi no son referenciadas.

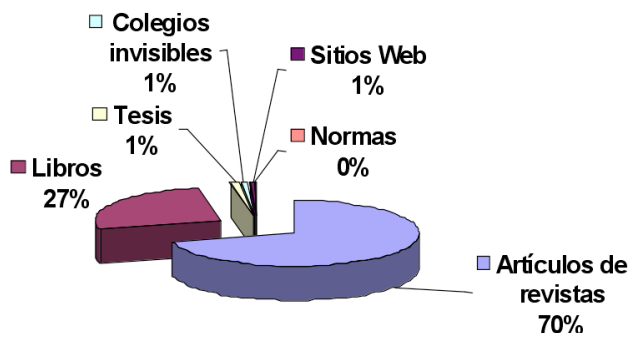

Figura 2. Tipo de referencias localizadas

La figura 3 se refiere a los libros. Para ello, se consultó LIBRUNAM con la finalidad de mostrar cuantos se localizaron dentro de este catálogo. Se constató que más del $50 \%$ (232) es material que ha sido adquirido por alguna biblioteca de la 
UNAM. Un porcentaje de material que fue consultado se encuentra en la Biblioteca IIMAS (102), y 171 referencias no se localizaron en la colección de la UNAM.
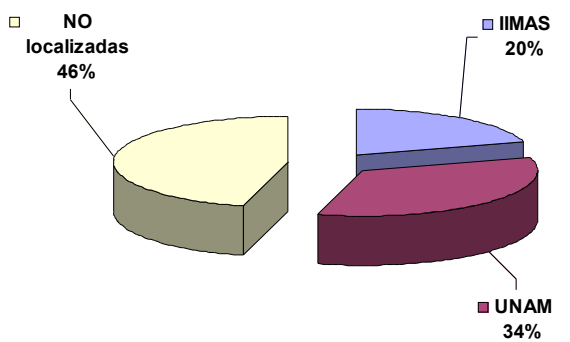

Figura 3. Referencias en libros

En relación a la figura anterior, surge el interrogante de por qué el $46 \%$ es material no registrado, por lo qué se decide analizar su fecha de publicación obteniendo los siguientes resultados: Para el año de 2007 solamente se hizo referencia a un libro -7 libros editados en los años de 2006 y 2004—; 8 y 9 para el 2005 y 2006 respectivamente; 10 libros en los años de 2000 y 2003; y 11 materiales para el 2002. Los anteriores a 2000 son 169 títulos por lo que se concluye con este apartado que el material que no es actual no se solicita para su compra o, si es solicitado pero difícil de adquirir.

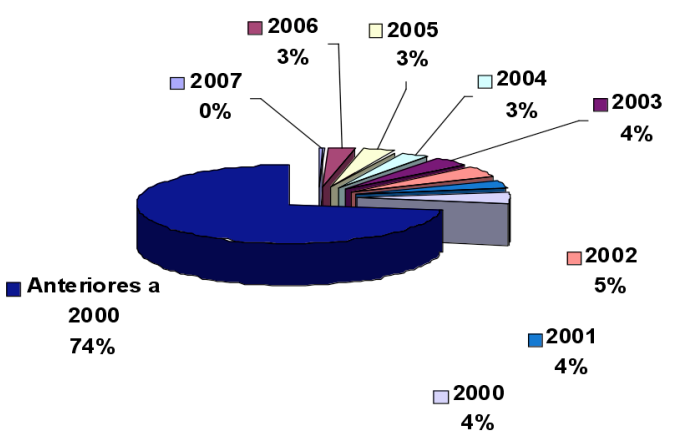

Figura 4. Análisis de las referencias de libros no localizadas

La figura 5 es demostrativa para ver las procedencias de las tesis que fueron referenciadas. Como se puede ver, la mayoría son de universidades extranjeras.

Las siguientes figuras son dedicadas exclusivamente a artículos en revistas ya que las publicaciones periódicas tienen un valor muy importante para la investigación por contener información más actualizada, por lo que su consulta es mayor.

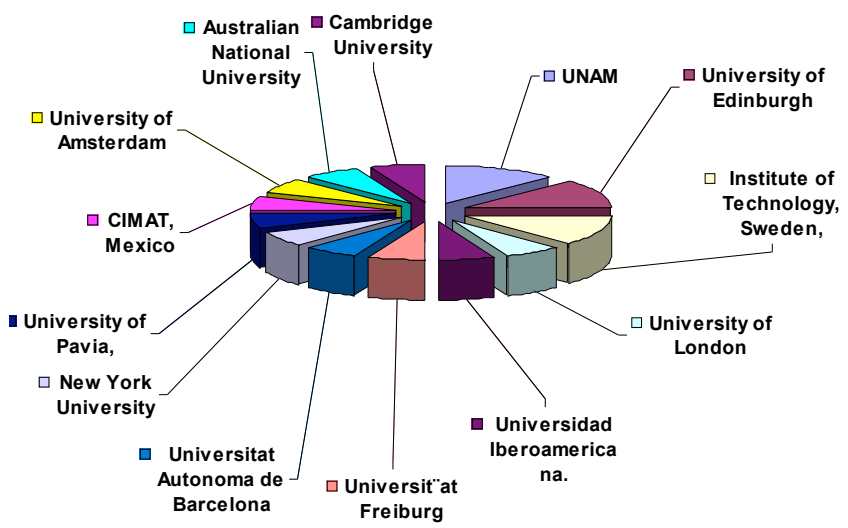

Figura 5. Procedencia de las tesis referenciadas

La figura 6 muestra el total de artículos en revistas que fueron referenciadas en los 67 artículos analizados, obteniendo 1106. Es esta parte, el análisis consistió en verificar que se encontraran registrados en el catálogo colectivo SERIUNAM: 722 lo fueron, y 384 no se encontraron.

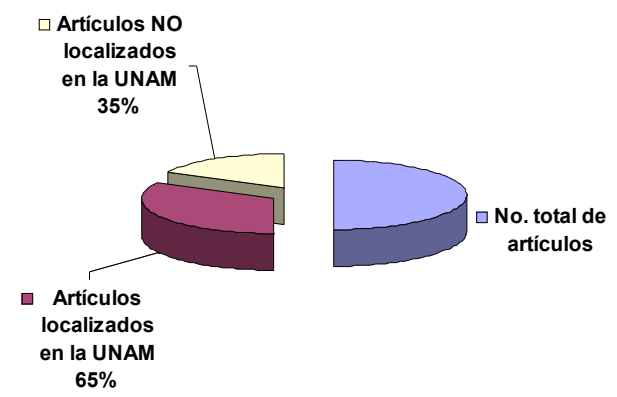

Figura 6. Artículos en revistas

En la siguiente figura se analizan los 722 artículos localizados. El $62 \%$ de éstos (441) son artículos que se localizan en el Sistema Bibliotecario; y el restante $38 \%$ son parte del acervo de la Biblioteca IIMAS, esto es 281 artículos.

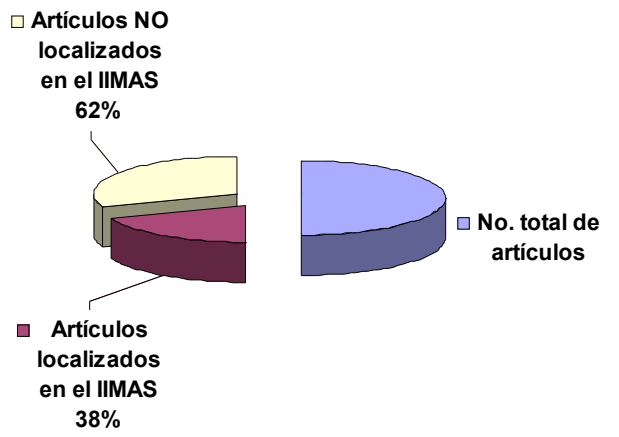

Figura 7. Procedencia de los artículos en revistas de Investigaciones en Matemáticas Aplicadas y en Sistemas. // Ibersid. (2008) 123-128. ISSN 1888-0967. 
La revista electrónica ha cobrado fuerza durante los últimos años y la UNAM también a incursionado en este rubro. La siguiente figura muestra el total de artículos localizados en este formato. Como se puede ver, de la cifra de 1106 artículos en revistas, 614 se tienen en formato electrónico, ya sea directamente por el editor o por un agregador de bases de datos. El resultado muestra que a menos de la mitad no se tiene acceso. Esto se puede deber a dos variables: 1) no se ha contratado la revista, 2) no se tiene acceso al volumen o número concreto.

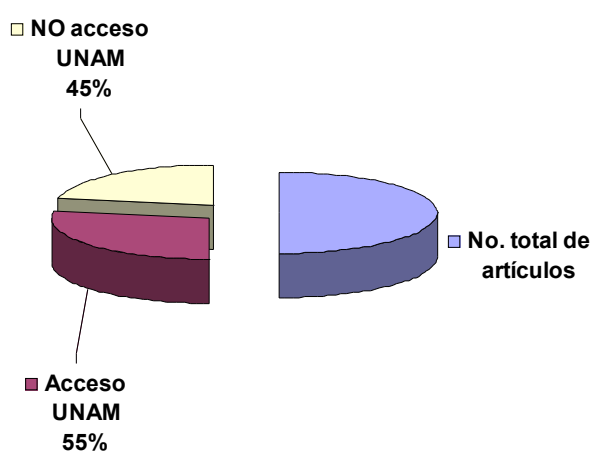

Figura 8. Revistas en formato electrónico

\section{Conclusiones}

Finalmente se concluye que el análisis realizado refleja que el material que se adquiere si cumple con las necesidades de información del IIMAS. Por la tanto, se enuncian las siguientes aseveraciones:

- Las revistas o publicaciones periódicas son importantes para tener información actualizada, por lo que su consulta es mayor que la de cualquier otro material. Por lo tanto, las revistas electrónicas también están jugando un papel fundamental y se tiene acceso a ellas a través de las colecciones de la Universidad.

- Los libros que se consultan se encuentran disponibles dentro de la Biblioteca IIMAS o bien dentro del Sistema Bibliotecario, por lo que se cumple el objetivo de tener más del $50 \%$ de la información. Otro aspecto importante que se remarca es el año de inicio de la revista, ya que cuanto más retrospectivo es, mayor es la consulta.

- La adquisición de libros de ediciones anteriores es difícil, por lo que la función del Sistema Bibliotecario es fundamental para el IIMAS y, pos supuesto, para la universidad.

- Las tesis son poco referenciadas. Quizá en un futuro está situación cambie ya que actual- mente la UNAM esta trabajando en el catálogo de tesis con acceso al texto completo.

- Otros materiales impresos se localizan a través de contactos personales, como son los colegios invisibles, por lo que el acceso es entre colegas.

Tener la certeza de dónde se localiza el material que los usuarios potenciales de la Biblioteca IIMAS consultan para la realización de sus artículos, pone de manifiesto que la colección es dinámica, y que no necesariamente todo lo que se adquiere es para ser citado; lo que da pie a analizar otras publicaciones, ya que en este estudio solamente se analizaron los artículos en revistas arbitradas.

\section{Referencias}

BIDI (Biblioteca Digital de la UNAM). http://www.dgb. unam.mx (2008-05, 2008-04).

Blanca, San José Montano and Manuel, Espantaleón Agreda and Ana de, Santiago Puchol and Victoria, Diaz Galcerán (2007). ¿Que utilizan nuestros usuarios investigadores hospitalarios? : Evaluación de la colección en cuatro bibliotecas hospitalarias basado en el análisis de citas bibliográficas de la producción científica de una especialidad medica http://eprints.rclis.org/archive/0001 1762/ (2008-04-22).

Boza Puerta, Mariano Gaspar Olmedo Granados (1998). ¿Se adaptan los fondos de mi biblioteca a las necesidades de los usuarios? : Un método objetivo de evaluación de colecciones en una biblioteca científica. http://fesabid98.florida-uni.es/Comunicaciones/m_boza/ m_boza.htm (2008-04-22).

Contreras Angeles, Laura (2006). Análisis de la visibilidad internacional de la literatura científica producida por investigadores mexicanos 1990-2004. México: El autor, 2006. $105 \mathrm{~h}$. Tesis licenciatura.

Franco Gómez, Yesenia (2006). Estudio bibliométrico de las referencias bibliográficas contenidas en la literatura del Departamento de Matemática Educativa del CINVESTAV-IPN: 1975-2004. México: El autor, 2006. 131 h. Tesis licenciatura.

Hemmings, Brian; Rushbrook, Meter; Smith Erica (2007). Academics'views on publishing refereed works : a content analysis. // Higher Education. ISSN 0882-4126. 54:2 (Agosto) 307-332.

Informe de actividades 2007 (2007). México : Instituto de Investigaciones en Matemáticas Aplicadas y en Sistemas, 2008. $276 \mathrm{P}$

Instituto de Investigaciones en Matemáticas Aplicadas y en Sistemas. Biblioteca. http://www.iimas.unam.mx (200805, 2008-04).

LIBRUNAM (Catálogo de libros de la UNAM) http://www.dgb.unam.mx (2008-05, 2008-04).

Mesquita, Rosa M. A. (2004). Estudo de citaçoes de documentos eletrônicos On-line em revistas da Area de Comunicaçao : Citation of On-line electronic documents by journals of the communication field. // Questao: revista da Faculdade de Biblioteconomia e Comunicaçao da UFRGS. ISSN 1807-8893. 10:2 (2004) 261-274

Perpiñá Galván, Juana; López Coig, M ${ }^{a}$. Luisa; Richart Martínez, Miguel; Cabrero García, Julio (1994). Análisis de referencias bibliográficas de artículos originales publicados en Enfermería Intensiva frente a tres revistas 
de ámbito general. // 5as. Jornadas de Información y Documentación de ciencias de la Salud. Palma de Mallorca 4 al 6, de mayo 1994 http://www.abadib.es/ 5jornadas-salud/comunicacion9.htm (2008-04-22).

Pinheiro Machado Mueller, Suzana (2002). What are third world scientific journals good for? : a case study on brazilian economic journals. I/: Simposio Internacional, investigación sobre la Comunicación Científica. Memorias México: UNAM, Centro Universitario de Investigaciones Bibliotecológicas; Facultad de Psicología. 93109.

SERIUNAM (Catálogo colectivo de publicaciones seriadas a nivel nacional) http://www.dgb.unam.mx (2008-05, 200804).

TESIUNAM (Catálogo de tesis de la UNAM). http:// www.dgb.unam.mx (2008-05, 2008-04).

Van Rooi, Hazel, Snyman, Retha (2006). A content analysis of literature regarding knowledge management opportunities for librarians. // Aslib Proceedings. ISSN 0001253X. 58:3 (2006)261-271.

White, Marilyn Domas, Marsh, Emily E (2003). Content analysis : a flexible methodology. // Library Trends. ISSN 0024-2594. 13:1. (2003) 20-26. de Investigaciones en Matemáticas Aplicadas y en Sistemas. // Ibersid. (2008) 123-128. ISSN 1888-0967. 